3889

\title{
Five years of EVLP: a monocentric experience
}

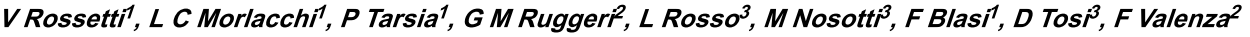

${ }^{1}$ Department of Pathophysiology and Transplantation, IRCCS Fondazione Ca' Granda Ospedale Maggiore Policlinico di Milano; Università degli Studi di Milano, Milano, Italy ${ }^{2}$ Dipartimento di Anestesia Rianimazione (Intensiva e Subintensiva) e Terapia del dolore, IRCCS Fondazione Ca' Granda Ospedale Maggiore Policlinico di Milano, Milano, Italy

${ }^{3}$ U.O. Chirurgia Toracica e dei Trapianti di Polmone, IRCCS Fondazione Ca' Granda Ospedale Maggiore Policlinico di Milano; Università degli Studi di Milano, Milano, Italy

Introduction : Ex Vivo Lung Perfusion (EVLP) is a valuable tool for the reassessment of marginal lungs before transplantation and could increase the pool of organs available; the aim of this study was to investigate the characteristics of the recipients of EVLP-assessed grafts and their outcomes in our hospital.

Methods : A retrospective study was conducted including all lung transplant (LuTx) recipients from January 2011 to December 2015 . Two groups of patients were identified based on their graft: EVLP assessed (Group A) or not (Group B).

Results : From January 2011 to December 2015, a total of 101 LuTx were performed; of those, 15 grafts underwent EVLP reconditioning. Table 1 summarises our results.

Conclusion : EVLP grafts were given to more severe recipients at time of LuTx, possibly affecting early perioperative results. However the incidence of primary graft dysfunction (PGD) Grade 3 and other medium and long-term outcomes of EVLP were comparable to conventional transplantations. EVLP reconditioning may increase the number of available grafts, also for those patients whose conditions are too severe to wait for another donor.

Table 1 - Continuous data expressed as median (IQR) and categorical data as proportion.

\begin{tabular}{|c|c|c|c|c|}
\hline & Population (101pts) & Group A (15 pts) & Group B (86 pts) & Sig. $(p)$ \\
\hline Age, years & $45(40 ; 50)$ & $42(32 ; 64)$ & $46(40 ; 51)$ & ns \\
\hline ECMO bridge & $18(18)$ & $4(27)$ & $14(16)$ & ns \\
\hline LAS at time of LuTx & $41(37 ; 45)$ & $61(39 ; 83)$ & $39(36 ; 42)$ & 0.041 \\
\hline PGD3 & $41(41)$ & $6(40)$ & $35(41)$ & ns \\
\hline $\begin{array}{l}\text { Mortality within } 90 \text { days from } \\
\text { LuTx }\end{array}$ & $6(6)$ & $3(20)$ & $3(4)$ & 0.042 \\
\hline ALAD & $26(24)$ & $3(19)$ & $23(25)$ & ns \\
\hline CLAD & $17(16)$ & $3(19)$ & $14(15)$ & ns \\
\hline Survival, months & $43(37 ; 47)$ & $37(22 ; 51)$ & $44(38 ; 49)$ & ns \\
\hline
\end{tabular}

Abbreviations: $L A S$, lung allocation score; $A L A D$ and CLAD, acute and chronic lung allograft dysfunction

Session:

From donor lung selection and organ preservation to lung transplant outcome (Poster Discussion)

Date/Time:

Sunday, September 10, 2017 / 14:45-16:45

Room:

Amber $5+6$ (South)

Category:

Transplantation

Keywords:

Transplantation, Critically ill patients, Experimental approaches 